\title{
Editorial
}

Monatsschr Kinderheilkd 2013 · 161:508-509

DOI 10.1007/s00112-013-2934-3

Online publiziert: 26. Mai 2013

(c) Springer-Verlag Berlin Heidelberg 2013

Redaktion

F. Zepp, Mainz

B. Willershausen, Mainz

B. Koletzko' $\cdot$ K.-E. Bergmann ${ }^{2}$-H. Przyrembel ${ }^{2}$

${ }^{1}$ Dr. von Haunersches Kinderspital, Klinikum der Universität München

${ }^{2}$ Deutsche Akademie für Kinder- und Jugendmedizin (DAKJ), Berlin

\section{Prophylaktische \\ Fluoridgabe im Kindesalter}

\section{Empfehlungen der DGKJ (Deutsche Gesellschaft für Kinder- und Jugendmedizin e. V.) und der DAKJ (Deutsche Akademie für Kinder- und Jugendmedizin e. V.)}

Willershausen et al. [14] fassten in ihrer sehr guten Übersichtsarbeit aus zahnärztlicher Sicht wichtige Informationen zur Förderung der Zahngesundheit zusammen und gaben in ihrer Tabelle 1 Empfehlungen zum Gebrauch von Fluoriden. Im Folgenden möchten wir die davon in einigen Aspekten abweichenden, aktuellen Empfehlungen der DGKJ und der DAKJ wiedergeben, wie sie in einer aktuellen Leitlinie der AWMF (Arbeitsgemeinschaft der wissenschaftlichen medizinischen Fachgesellschaften, [4]) dargestellt sind.

\section{Zahnreinigung}

Kinder sollten im älteren Säuglings- und im Kleinkindalter in behutsamen Schritten spielerisch und in positiv erlebter Weise an die regelmäßige Zahnreinigung herangeführt werden, die sie mit zunehmendem Alter selbst durchführen sollen. Die Zahnreinigung kann im Säuglingsalter zunächst mit einem Wattestäbchen oder aber mit einer altersgerecht geformten Zahnbürste vorgenommen werden.

\section{Zahnpasta}

Zahnpasta ist ein kosmetisches Produkt zur Zahnreinigung, das nicht zum Verzehr bzw. zum Verschlucken vorgesehen ist. Das Bundesinstitut für Risikobe- wertung stellte fest, dass in Deutschland für Kinder angebotene Zahnpasten Substanzen enthalten, die als Inhaltsstoffe für Mundpflegemittel vorgesehen, aber nicht für den Verzehr geeignet sind. Für einige Inhaltsstoffe von Zahnpasten werden ausdrückliche Warnhinweise empfohlen wie Nicht verschlucken oder Übermäßiges Verschlucken vermeiden (bei Kinderzahnpasten z. B. enthaltene Fluoridverbindungen). Auch deshalb sollten keine Kinderzahnpasten eingesetzt werden, die durch Aufmachung bzw. für Kleinkinder attraktive Farben oder Geschmacksstoffe das kindliche Verschlucken fördern.

\) Ein Zahnpastafluoridgehalt $<550$ ppm zeigt keine nachgewiesene kariesprotektive Wirkung

Zahnpasten sollen erst dann regelmäßig verwendet werden, wenn das Kind sie nach dem Zähneputzen weitgehend ausspucken kann. Bei großer interindividueller Variation ist diese Fähigkeit in der Regel im Alter von 4 Jahren (5. Lebensjahr) erreicht. Die Empfehlung der Anwendung einer geringen Menge (dünner Film, erbsengroße Menge) ist auf eine kariesprophylaktische Wirksamkeit nicht untersucht, wird in der Praxis nicht richtig umgesetzt und bietet keinen hinrei- 
Tab. 1 Pädiatrische Empfehlungen für den Gebrauch von Fluoriden

\begin{tabular}{|c|c|c|c|c|}
\hline Alter (Jahre) & 0 & 2 & 4 & 6 \\
\hline $\begin{array}{l}\text { Basisprophy- } \\
\text { laxe: Fluroidta- } \\
\text { blette }\end{array}$ & $\begin{array}{l}\text { 1-mal } \\
\text { täg- } \\
\text { lich }\end{array}$ & $\begin{array}{l}\text { 1-mal } \\
\text { täg- } \\
\text { lich }\end{array}$ & \multicolumn{2}{|c|}{$\begin{array}{l}\text { Kann entfallen, wenn Fluoridsalz und fluoridierte Zahnpasta } \\
\text { eingesetzt werden }\end{array}$} \\
\hline Fluoridsalz & \multicolumn{3}{|c|}{ Regelmäßige Verwendung } & \\
\hline $\begin{array}{l}\text { Fluoridzahn- } \\
\text { pasta }\end{array}$ & $\begin{array}{l}\text { Nicht e } \\
\text { fohlen }\end{array}$ & & $\begin{array}{l}\text { 2-mal täglich Zähneputzen mit } \\
\text { dünnem Film Zahnpasta mit } \\
>1000 \text { ppm Fluorid, sofern das } \\
\text { Kind zuverlässig ausspuckt }\end{array}$ & $\begin{array}{l}\text { 2-mal täglich Zähneput- } \\
\text { zen mit Erwachsenen- } \\
\text { zahnpasta (>1000 ppm } \\
\text { Fluorid) }\end{array}$ \\
\hline
\end{tabular}

Tab. 2 Empfohlene Tagesdosis der Fluo-

ridsupplementierung ${ }^{\mathrm{a}}$. (Nach [6])

\begin{tabular}{|ll}
\hline Alter (Jahre) & Fluorid $(\mathbf{m g} /$ Tag) \\
\hline 0 bis $<2$ & 0,25 \\
\hline$>2$ bis $<4$ & 0,5 \\
\hline$>4$ bis $<6$ & 0,75 \\
\hline$>6$ & 1,0 \\
\hline $\begin{array}{l}\text { aFür Kinder ohne weitere relevante Fluoridquellen } \\
\text { und bei einem Fluoridgehalt im Trinkwasser bis zu } \\
0,3 \text { mg/l }\end{array}$ \\
\hline
\end{tabular}

chenden Schutz vor dem Verschlucken größerer Mengen an Zahnpasta.

Eine rezente Cochrane-Metaanalyse zeigte bei Schulkindern und Jugendlichen bis zum Alter von 16 Jahren eine kariespräventive Wirkung der regelmäßigen Anwendung fluoridierter Zahnpasta mit einem Fluoridgehalt von 1000 1250 ppm (mittlere Kariesreduktion $23 \%$ ) und von $2400-2800$ ppm (mittlere Kariesreduktion 36\%), während Zahnpasten mit einem Fluoridgehalt von 440 550 ppm mit keiner Kariesreduktion einhergingen [12]. Somit besteht keine Grundlage für eine Empfehlung der Anwendung der in Deutschland üblichen Kinderzahnpasten mit einem Fluoridgehalt von 500 ppm oder für die Anwendung fluoridierter Zahnpasta im Vor schulalter. Die tägliche Anwendung einer Zahnpasta mit mindestens 1000 ppm Fluorid wird im Kleinkindalter wegen des Risikos einer übermäßigen Fluoridexposition und einer Fluorose nicht empfohlen; sie kann eingesetzt werden, wenn das Kind Zahnpasta zuverlässig ausspuckt (• Tab. 1).

\section{Fluoridsupplemente}

Fluoride wirken sowohl bei topischer als auch bei systemischer Anwendung kariespräventiv [2, 5, 6]. Während der Zahnentwicklung wird Fluorid aus dem Kreislauf in Zahnschmelz und Dentin eingebaut. Fluorohydoxyapatit zeigt eine stärkere Säureresistenz als Hydroxyapatit. Nach dem Zahndurchbruch wirkt oral zugeführtes Fluorid durch Schmelzkontakt während des Konsums, durch Ausscheidung mit dem Speichel und durch Aufnahme in Biofilme auf der Zahnoberfläche antikariös. Ein maximaler kariespräventiver Effekt wird durch die kombinierte prä- und posteruptive Fluoridexposition erzielt [10].

Eine jüngere Cochrane-Metaanalyse zeigte bei Kindern und Jugendlichen bis zum Alter von 16 Jahren durch die Anwendung von Fluoridsupplementen eine Kariesreduktion um $24 \%$ bei bleibenden Zähnen, während die Wirkung bei Milchzähnen unklar blieb [11]. Hierbei ist zu berücksichtigen, dass die klassischen kontrollierten, z. T. auch doppelblind durchgeführten Studien zur Wirksamkeit von Fluoridsupplementen auf die Milchzahnkaries in dieser Cochrane-Analyse nicht vollumfänglich berücksichtigt wurden [1, $3,7,8,9,13]$.

Bis zum Erreichen einer regelmäßigen Fluoridexposition in angemessener Menge durch größere Verzehrsmengen fluoridangereicherten Speisesalzes und durch fluoridierte Zahnpasta wird im Säuglingsund Kleinkindalter die tägliche Zufuhr eines Fluoridsupplements empfohlen. Die empfohlene Tagesdosis unter Standardbedingungen zeigt $\bullet$ Tab. 2. Bis zum 2. erlebten Frühsommer, d. h. je nach Geburtszeitpunkt für die ersten 12 bis $18 \mathrm{Mo-}$ nate, soll die Fluoridgabe mit täglich 400500 I.U. Vitamin D kombiniert werden.

\section{Korrespondenzadresse}

\section{Prof. Dr. B. Koletzko}

Dr. von Haunersches Kinderspital, Klinikum der Universität München

Lindwurmstraße 4, 80337 München

office.koletzko@med.uni-muenchen.de

\section{Literatur}

1. Aasenden R, Peebles TC (1974) Effects of fluoride supplementation from birth on human deciduous and permanent teeth. Arch Oral Biol 19:321-326

2. American Academy of Pediatrics Committee on Nutrition (1995) Fluoride supplementation for children: interim policy recommendations. Pediatrics 95(5):777

3. Andersson R, Grahnén H (1976) Fluoride tablets in pre-school age - effect on primary and permanent teeth. Swed Dent J 69:137-143

4. Arbeitsgemeinschaft der wissenschaftlichen medizinischen Fachgesellschaften (AWMF) (2013) S2kLeitlinie Fluoridierungsmaßnahmen zur Kariesprophylaxe. AWMF-Register Nr. 083-001. AWMF, Düsseldorf, http://www.awmf.org/uploads/tx_szleitlinien/083-001I_S2k_Fluoridierungsmaßnahmen_ zur_Kariesprophylaxe_2013-01.pdf. Zugegriffen: 03.05.2013

5. Buzalaf MA, Pessan JP, Honório HM, ten Cate JM (2011) Mechanisms of action of fluoride for caries control. Monogr Oral Sci 23:97-114

6. Deutsche Akademie für Kinder- und Jugendmedizin (2007) Empfehlungen zur Prävention der Milchzahnkaries. Monatsschr Kinderheilkd 155:544-548

7. Fanning EA, Cellier KM, Leadbeater MM, Somerville CM (1975) South Australian kindergarten children: fluoride tablet supplements and dental caries. Aust Dent J 20:7-9

8. Hamberg L (1971) Controlled trial of fluoride in vitamin drops for prevention of caries in children. Lancet 1:441-442

9. Margolis FJ, Reames HR, Freshman E et al (1975) Fluoride. Ten-year prospective study of deciduous and permanent dentition. Am J Dis Child 129:794800

10. Singh KA, Spencer AJ, Armfield JM (2003) Relative effects of pre- and posteruption water fluoride on caries experience of permanent first molars. J Public Health Dent 63:11-19

11. Tubert-Jeannin $S$, Auclair $C$, Amsallem E, Tramini $P$ et al (2011) Fluoride supplements (tablets, drops, lozenges or chewing gums) for preventing dental caries in children. Cochrane Database Syst Rev 12:CD007592

12. Walsh T, Worthington HV, Glenny AM et al (2010) Fluoride toothpastes of different concentrations for preventing dental caries in children and adolescents. Cochrane Database Syst Rev 1:CD007868

13. Widenheim J, Birkhed D (1991) Caries preventive effect on primary and permanent teeth and cost effectiveness of an NaF tablet pre-school program. Community Dent Oral Epidemiol 19:88-92

14. Willershausen I, Lampe F, Fink O (2013) Grundlagen der Zahngesundheit: Erhaltung und Prävention. Monatsschr Kinderheilkd 161. DOI 10.1007/ s00112-012-2832-0 